\title{
Unexpected Mycoplasma hominis infection in two renal transplant recipients traced back to the same donor by whole-genome sequencing
}

\author{
V. Hinić ${ }^{1}$ • H. M. B. Seth-Smith ${ }^{1,2} \cdot$ S. Damm ${ }^{3} \cdot$ P. Amico ${ }^{3}$ N N. Khanna ${ }^{4} \cdot$ A. Egli ${ }^{1,2}$ • V. Bättig ${ }^{4}$
}

Received: 30 September 2020 / Accepted: 25 November 2020 / Published online: 23 December 2020

(C) The Author(s) 2020

\begin{abstract}
Mycoplasma hominis is a common colonizer of the lower genitourinary tract. Although its clinical relevance for causing urogenital infections in immunocompetent individuals is controversial, this bacterium has been involved in severe invasive infections in allograft recipients. In this report, we describe two cases of $M$. hominis infection in two young renal transplant recipients within the first month post-transplant. Although at first no epidemiological link between the two cases had been suspected, whole-genome sequencing (WGS) analysis showed that both isolates were identical, highly suggestive of an origin with the common organ donor.
\end{abstract}

Keywords Mycoplasma hominis $\cdot$ Renal transplant · Typing $\cdot$ Whole-genome sequencing (WGS)

\section{Introduction}

Mycoplasma hominis belong to the class of Mollicutes, are the smallest self-replicating prokaryotes, and are characterized by the absence of a cell wall. Since M. hominis is frequently detected in the lower urogenital tract in sexually active healthy men and women, the understanding of their etiologic role as a pathogen is somewhat controversial [1]. Although infections in immunocompetent patients have been reported [2,3], most publications implicate $M$. hominis in invasive disease in immunosuppressed persons [4-8].

In contrast to other Mycoplasma spp., M. hominis can grow on conventional bacteriological media. However, the growth occurs often only after prolonged incubation, and, due to their

\section{Hinić}

vladimira.hinic@usb.ch

1 Division of Clinical Bacteriology and Mycology, University Hospital Basel, Basel, Switzerland

2 Applied Microbiology Research, Department of Biomedicine, University of Basel, Basel, Switzerland

3 Clinic for Transplantation Immunology and Nephrology, University Hospital Basel, Basel, Switzerland

4 Division of Infectious Diseases and Hospital Epidemiology, University Hospital Basel, Basel, Switzerland morphology, the fine colonies can be easily overlooked especially in the presence of concomitant contaminating flora.

In this report, we describe two cases of extragenital infection with M. hominis in two renal transplant recipients, discovered accidentally in the bacterial culture. The epidemiological link between the two cases has been established by whole-genome sequence (WGS) analysis.

\section{Case 1}

A 19-year-old patient with history of diethylene glycol poisoning-induced end-stage renal disease underwent kidney transplantation from a deceased donor. Three days later, a relaparotomy was performed because of postoperative bleeding with consecutive retroperitoneal hematoma. Due to persistent fever and elevated inflammation markers, antibiotic treatment with piperacillin-tazobactam was started with inadequate response. A computed tomography (CT) scan of abdomen and pelvis 2 weeks after the transplantation revealed progredient subcutaneous epifascial fluid collection along the surgical suture, compatible with postoperative residual hematoseroma with possible secondary infection. The collection was drained and an aspirate sent to the microbiology laboratory for bacterial culture. Direct Gram stain revealed copious leukocytes and erythrocytes, but no bacteria. On the sixth day of incubation, 
small translucent colonies on Brucella agar with 5\% horse blood (BD) incubated under anaerobic atmosphere were observed (Fig. 1, isolate 612321-19). 16S rRNA gene sequencing identified the bacteria as $M$. hominis. Upon receipt of microbiology result, the antimicrobial therapy was changed to doxycycline $100 \mathrm{mg}$ twice daily per os for 7 days. All blood cultures and urine samples remained without growth.

Under adequate treatment, inflammation parameters completely normalized and the patient was discharged home. In the last outpatient consultation 6 months after the transplantation, the patient was well with no sign of infection and the kidney function was stable at an eGFR of $71 \mathrm{ml} / \mathrm{min} / 1.73 \mathrm{~m}^{2}$ calculated by CKD-EPI (Chronic Kidney Diesaese- Epidemiology Collaboration) without proteinuria. See timeline in Fig. 2.

\section{Case 2}

A 26-year-old man received a kidney transplantation from a deceased donor due to tubulointerstitial nephropathy of unknown etiology. On day two post-transplantation, the patient developed clinical signs of ileus with acute abdominal pain and emesis. CT scan indicated a pneumoperitoneum, raising suspicion about intestine perforation. On the same day, explorative laparotomy was performed, whereby organ perforation could be excluded, so that the pneumoperitoneum seen was associated with the peritoneal dialysis. A deep intraabdominal swab from the laparotomy site and blood cultures were collected and showed no bacterial growth. Due to diffuse hemorrhage originating from the surgical wound, a further laparotomy with evacuation of a hematoma in the abdominal wall was necessary 2 days later. At day 26 after transplantation, the patient was discharged home. In the first outpatient consultation a few days later, a wound dehiscence was seen with consecutive operative revision once again and fascial reclosure. Intraoperatively, two

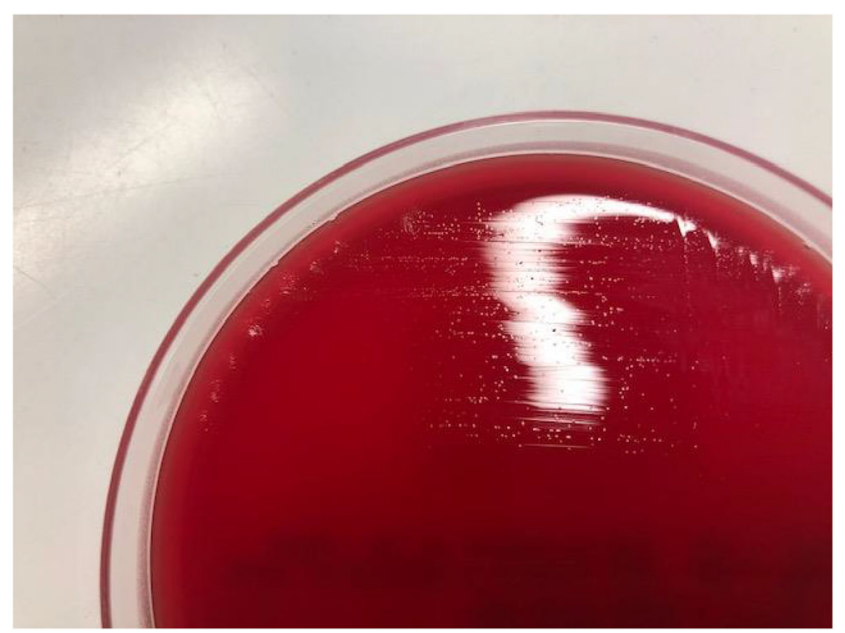

Fig. 1 Growth of M. hominis. Picture was taken after 6 days of incubation on Brucella agar under anaerobic conditions deep intraabdominal swabs were obtained. Direct Gram stain was performed but showed no presence of bacteria. On the fifth day of incubation, tiny, translucent colonies were observed on Brucella agar with 5\% horse blood incubated under anaerobic atmosphere (isolate 613095-19). Again, 16S rRNA gene sequencing identified the isolate as M. hominis. Accordingly, doxycycline $100 \mathrm{mg}$ twice daily was initiated for 7 days with excellent clinical response. In the last outpatient consultation 6 months after the transplantation, the patient was well with no sign of infection and the kidney function was stable at an eGFR of $61 \mathrm{ml} / \mathrm{min} / 1.73 \mathrm{~m}^{2}$ calculated by CKD-EPI without proteinuria. See timeline in Fig. 2.

\section{Microbiological and whole-genome sequencing analysis}

Intraoperative specimens from both patients were processed routinely at the microbiology laboratory including Gram stain and culture on Columbia agar with 5\% sheep blood (BD) and Chocolate agar PolyViteX (bioMérieux) incubated aerobically for 2 days, fluid thioglycolate medium (BD) incubated aerobically for 6 days, and Brucella agar with 5\% horse blood (BD) incubated for 6 days under anaerobic conditions. Aerobic and anaerobic blood cultures obtained from both patients were incubated for 5 days in a BacT/ALERT ${ }^{\circledR}$ VIRTUO ${ }^{\circledR}$ detection system (bioMérieux). 16S rRNA gene sequencing was performed as described previously [9]. The two case strains were isolated within 16 days of each other. DNA from both isolates, and an unrelated $M$. hominis isolate from the same time period as a control (404665-19), was extracted by Qiagen EZ1. WGS was performed on an Illumina NextSeq500 2x150bp after Nexteraflex library preparation, providing mean coverage over $69 \times$. An ad hoc core genome MLST (cgMLST) scheme based on the ATCC 23114 genome (FP236530) and 16 further query genomes (Table S1) was calculated in Ridom Seqsphere+ v 6.0.2 (https://www.ridom.de/seqsphere/u/Tutorial_for_Ad_ hoc_cgMLST_Schema.html) producing a scheme with 413 targets (Table S2). cgMLST analysis (Fig. 3) and further complete genome SNP analysis in CLC Genomics Workbench v20. 0.2 using the genome of isolate 612321-20 as the reference (data not shown) show that the two case isolates are identical, in contrast to the control isolate and further database isolates. All NGS data has been deposited under project PRJEB40931.

\section{Discussion}

Mycoplasma hominis has been reported as a cause of serious infections in allograft recipient patients, mostly kidney recipients $[4,6-8,10]$. The incidence of infections may even be underrecognized, due to difficulties in detection of this bacterial group with conventional microbiological diagnostic 
Fig. 2 Timeline for case 1 and case 2
Case 1: 19 years, end-stage renal disease due to diethylene glycol poisoning

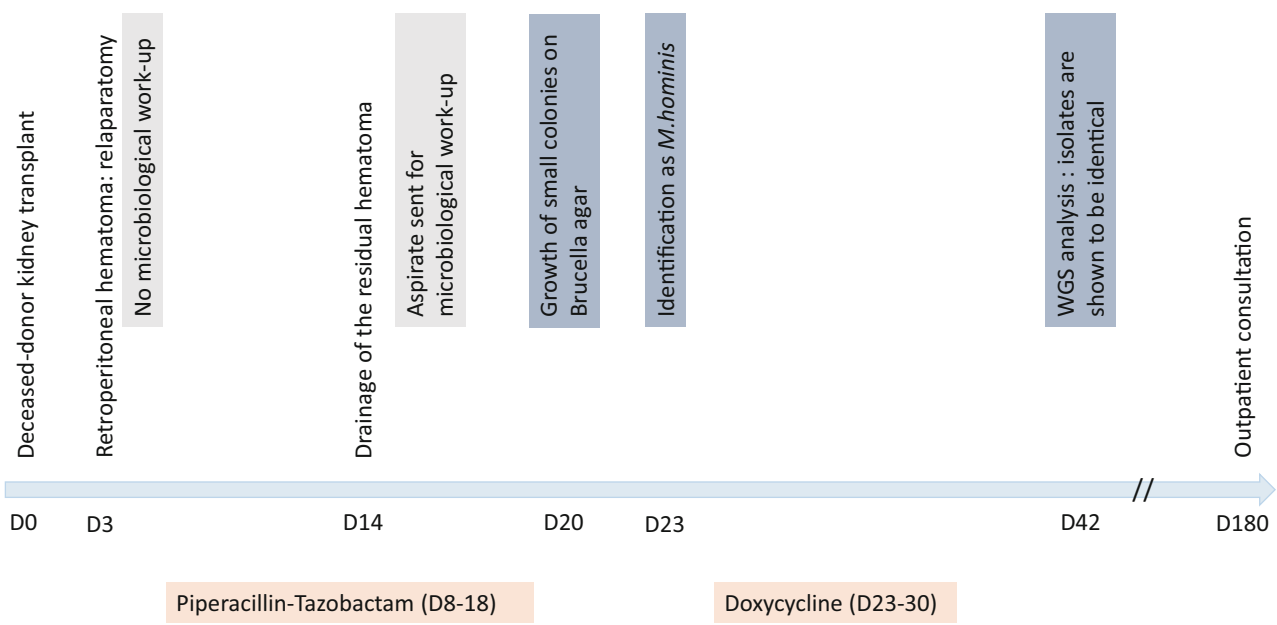

Case 2: 26 years, end-stage renal disease due to tubulointerstitial nephropathy of unknown etiology

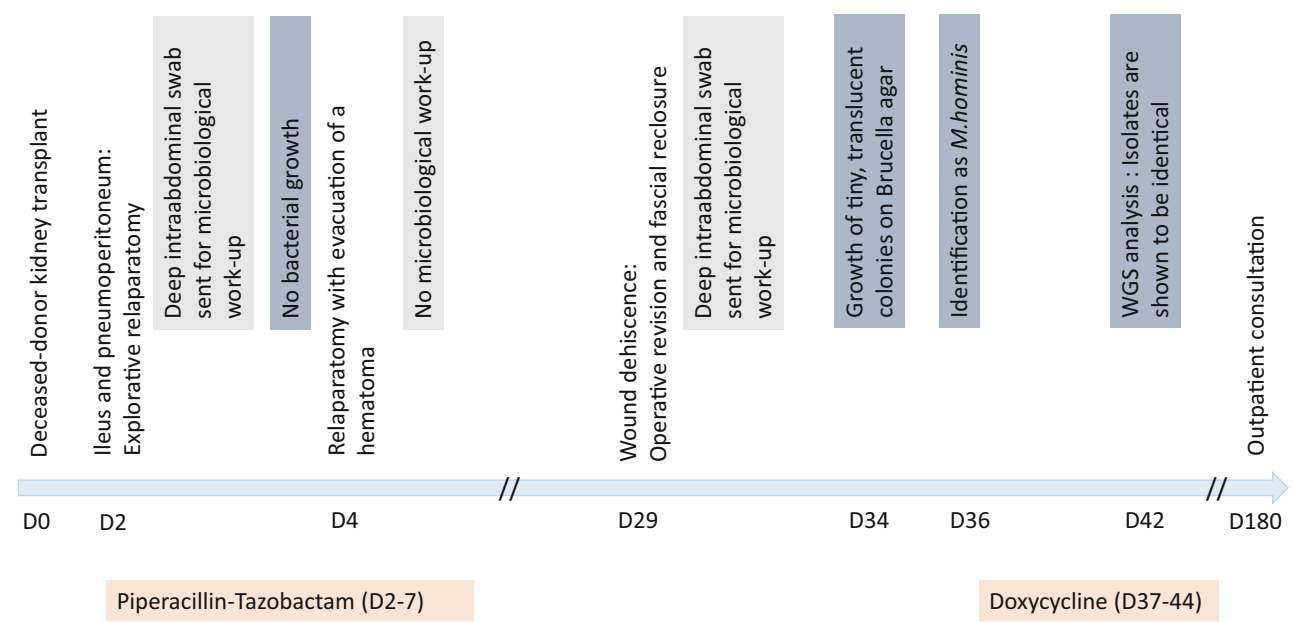

procedures. Culturing Mycoplasma spp. is laborious, usually requiring special growth media, and, depending on species, can take up to several weeks $[11,12]$. M. hominis is the only pathogenic Mycoplasma of human origin that can grow on routinely used bacteriologic blood-based media in both aerobic and anaerobic conditions and for which growth is usually observed in 2 to 6 days from primary specimens. Still, they are often difficult to find in the routine culture due to the following reasons: (a) the organism is never recognized in the direct Gram stain, since there is no cell wall; (b) the colonies are tiny and translucent, and can be mistaken for material (fat droplets from the tissue); and (c) colony growth is visible not before $48 \mathrm{~h}$ of incubation. In both cases described here, growth was observed only after 5 or 6 days of incubation. This was only possible as our laboratory routinely incubates anaerobic plates from intraoperative samples from primary sterile sites for longer period of 6 days (in contrast, aerobic cultures are incubated for 2 days); in most laboratories, both aerobic and anaerobic cultures are incubated for only 2 days. Additionally, Mycoplasma colonies can be easily overlooked if overgrown by other microbiota present in the sample. Therefore, detection of M. hominis should not normally be relied upon in routine bacteriological culture.

Blood cultures collected from both patients were negative for growth after 5 days of incubation. This does not rule out bloodstream infection: automated blood culture systems often fail to detect growth of $M$. hominis. This failure is not completely understood, but might be attributed to the following: (a) BacT/ALERT (bioMérieux) and BACTEC (BD) blood cultures contain anticoagulant sodium polyanethol sulfonate, which may inhibit Mycoplasma, and therefore, the growth may not exceed the threshold value for positivity, resulting in the negative blood culture $[13,14]$, or (b) despite growth in the blood culture, the unique metabolic properties of $M$. hominis create very low amounts of $\mathrm{CO}_{2}$ which are insufficient to trigger the automated blood culture system [13]. As such, molecular methods are most reliable for $M$. hominis detection. Several in-house real-time PCR systems $[15,16]$, and some commercial systems [17], have been developed, showing increased sensitivity and specificity of $M$. hominis detection in clinical specimens in comparison to culture. 


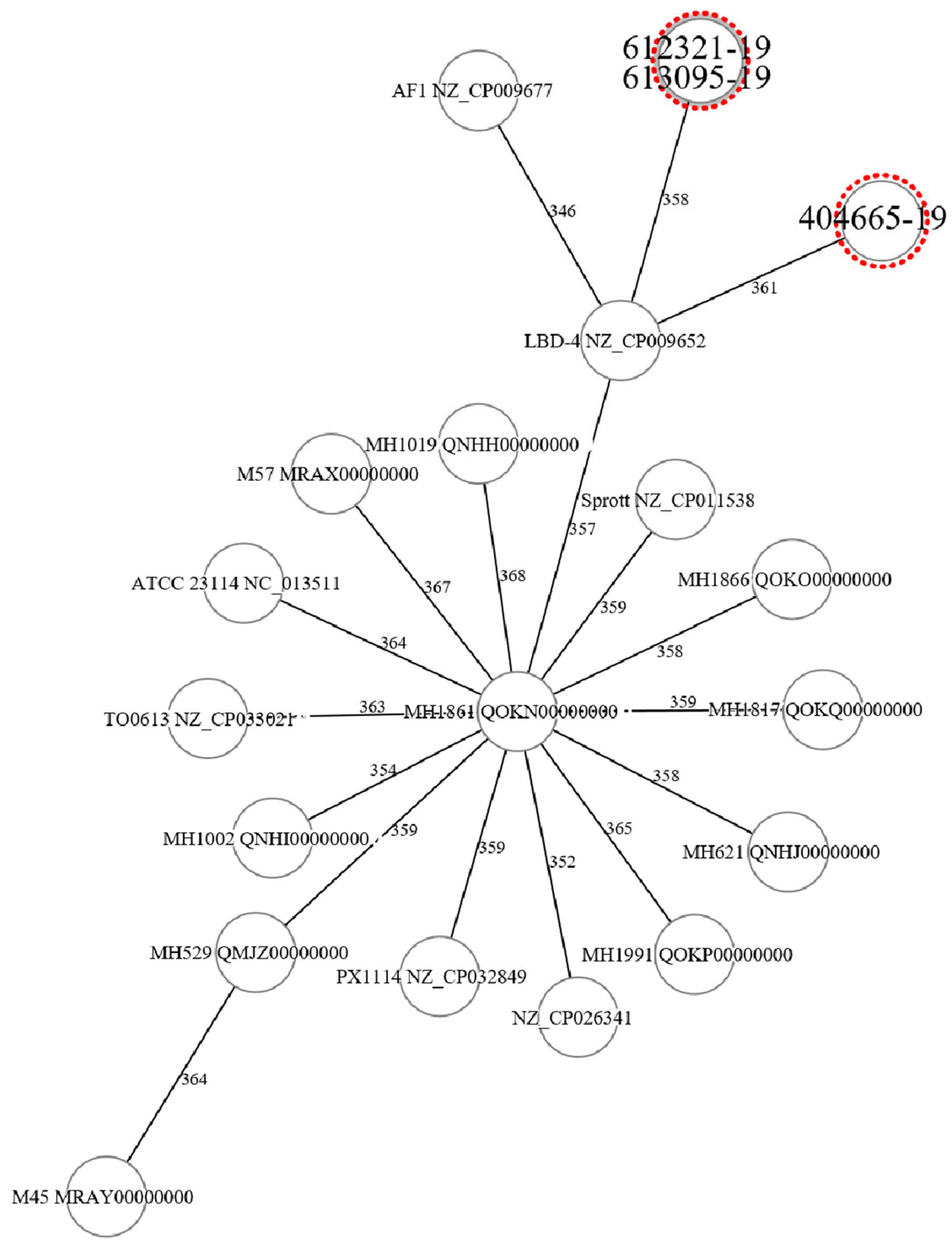

Fig. 3 Minimum spanning tree based on cgMLST analysis of M. hominis genomes. Figure was generated in Ridom Seqsphere+ based on an ad hoc scheme using 413 targets. Distances between genomes show the number

The growth of $M$. hominis, usually found in urogenital samples, in two different intraoperative samples within a very brief time frame of only 2 weeks was very unusual. For this reason, we initiated further investigation including WGStyping of both $M$. hominis isolates. WGS provides the high of allele differences. The two case isolates are 612321-19 and 613095-19, and are seen to be identical, and the control isolate is 404665-19. Other genomes from databases are shown for context

resolution for epidemiological investigations [18] and has previously been used in an epidemiological investigation of M. hominis [19]. In our case report, the results of the cgMLST analysis shows that isolates from patient 1 and patient 2 were identical, highly suggestive of a common source 
(Fig. 3). Therefore, the infection is likely to have originated from the organ donor. Unfortunately, no clinical data on the colonization/infection status of the donor were available. The main risk factor for infection in both patients was probably the immunosuppression. A liver transplantation from the same deceased donor was performed without any postoperative complications, and we therefore hypothesize that the donor was colonized/infected urogenitally. The possibility of Mycoplasma infection in allograft recipients should be raised among the clinicians, especially if cultures appear negative, and there is no improvement under broad-spectrum beta-lactam antimicrobial therapy.

Serological screening for certain infectious agents in deceased organ and living kidney donors is mandatory. Depending on clinical circumstances, additional microbiological cultures are sometimes required, but they do not routinely include screening for urogenital Mollicutes. The prevalence of $M$. hominis in sexually active males can be as high as $20 \%$, and $3.1-15 \%$ in females, depending on methodological and true population differences [1]. Although cases of non-sexual transmission have been described [20], most cases of genital colonization with Mollicutes among adults occur as a result of sexual contact and are correlated with the number of sexual partners [21, $22]$. For this reason, screening of young, sexually active deceased and living kidney donors could help prevent immunosuppression-associated post-transplant infections with these facultative pathogens. Gerber and colleagues suggest screening of renal allograft recipients for urogenital Mycoplasma spp. and Ureaplasma spp. in symptomatic urinary tract infections including pyelonephritis with negative standard urine cultures, signs or symptoms of urethritis, graft dysfunction in a patient with concomitant sterile leukocyturia, or deep wound infections with negative or unconvincing standard cultures [4]. Neither of our patients was screened urogenitally for presence of $M$. hominis.

In conclusion, transplant patients with no improvement of febrile infection under broad-spectrum beta-lactam antibiotics with negative bacterial cultures should raise suspicion for infection with Mycoplasma or other Mollicutes. In such cases, a specific PCR from the intraoperative samples should be requested by the clinician. A general screening of the young, sexually active donors for genital Mollicutes is an issue that deserves further discussion, in particular if kidney transplantation is considered. WGS proved to be an excellent tool for epidemiologic investigations even for fastidious and slow-growing bacterial pathogens such as Mollicutes.

Supplementary Information The online version contains supplementary material available at https://doi.org/10.1007/s10096-020-04116-y.

Authors' contributions VH and VB wrote the manuscript, HMBSS provided WGS data, SD and PA provided additional clinical data, NK and
$\mathrm{AE}$ contributed methodological inputs and reviewed the manuscript critically.

Funding Open access funding provided by University of Basel.

Data availability Not applicable.

\section{Compliance with ethical standards}

Conflict of interest Not applicable.

Ethics approval Not applicable.

Consent to participate Not applicable.

Consent for publication Available.

Code availability Not applicable.

Open Access This article is licensed under a Creative Commons Attribution 4.0 International License, which permits use, sharing, adaptation, distribution and reproduction in any medium or format, as long as you give appropriate credit to the original author(s) and the source, provide a link to the Creative Commons licence, and indicate if changes were made. The images or other third party material in this article are included in the article's Creative Commons licence, unless indicated otherwise in a credit line to the material. If material is not included in the article's Creative Commons licence and your intended use is not permitted by statutory regulation or exceeds the permitted use, you will need to obtain permission directly from the copyright holder. To view a copy of this licence, visit http://creativecommons.org/licenses/by/4.0/.

\section{References}

1. Horner P, Donders G, Cusini M, Gomberg M, Jensen JS, Unemo M (2018) Should we be testing for urogenital Mycoplasma hominis, Ureaplasma parvum and Ureaplasma urealyticum in men and women?-a position statement from the European STI Guidelines Editorial Board. J Eur Acad Dermatol Venereol 32(11):1845-1851

2. Pascual A, Perez MH, Jaton K, Hafen G, Di Bernardo S, Cotting J et al (2010) Mycoplasma hominis necrotizing pleuropneumonia in a previously healthy adolescent. BMC Infect Dis 10:335

3. Mechai F, Le Moal G, Duchene S, Burucoa C, Godet C, Freslon M (2006) Mycoplasma hominis osteitis in an immunocompetent man. Eur J Clin Microbiol Infect Dis 25(11):715-717

4. Gerber L, Gaspert A, Braghetti A, Zwahlen H, Wuthrich R, Zbinden R et al (2018) Ureaplasma and Mycoplasma in kidney allograft recipients-a case series and review of the literature. Transpl Infect Dis 20(5):e12937

5. Sampath R, Patel R, Cunningham SA, Arif S, Daly RC, Badley AD et al (2017) Cardiothoracic transplant recipient Mycoplasma hominis: an uncommon infection with probable donor transmission. EBioMedicine. 19:84-90

6. Rohner P, Schnyder I, Ninet B, Schrenzel J, Lew D, Ramla T et al (2004) Severe Mycoplasma hominis infections in two renal transplant patients. Eur J Clin Microbiol Infect Dis 23(3):203-204

7. Pastural M, Audard V, Bralet MP, Remy P, Salomon L, Tankovic J et al (2002) Mycoplasma hominis infection in renal transplantation. Nephrol Dial Transplant 17(3):495-496 
8. Legg JM, Titus TT, Chambers I, Wilkinson R, Koerner RJ, Gould FK (2000) Hematoma infection with Mycoplasma hominis following transplant nephrectomy. Clin Microbiol Infect 6(11):619-621

9. Goldenberger D, Kunzli A, Vogt P, Zbinden R, Altwegg M (1997) Molecular diagnosis of bacterial endocarditis by broad-range PCR amplification and direct sequencing. J Clin Microbiol 35(11):27332739

10. Miranda C, Carazo C, Banon R, Mendoza J, Montes A, de la Rosa M (1990) Mycoplasma hominis infection in three renal transplant patients. Diagn Microbiol Infect Dis 13(4):329-331

11. Baseman JB, Cagle M, Korte JE, Herrera C, Rasmussen WG, Baseman JG et al (2004) Diagnostic assessment of Mycoplasma genitalium in culture-positive women. J Clin Microbiol 42(1): 203-211

12. Waites KB, Talkington DF (2004) Mycoplasma pneumoniae and its role as a human pathogen. Clin Microbiol Rev 17(4):697-728 table of contents

13. Waites KB, Canupp KC (2001) Evaluation of BacT/ALERT system for detection of Mycoplasma hominis in simulated blood cultures. J Clin Microbiol 39(12):4328-4331

14. Waites KB, Katz B, Schelonka RL (2005) Mycoplasmas and ureaplasmas as neonatal pathogens. Clin Microbiol Rev 18(4): 757-789

15. Ferandon C, Peuchant O, Janis C, Benard A, Renaudin H, Pereyre S et al (2011) Development of a real-time PCR targeting the yidC gene for the detection of Mycoplasma hominis and comparison with quantitative culture. Clin Microbiol Infect 17(2):155-159
16. Pascual A, Jaton K, Ninet B, Bille J, Greub G (2010) New diagnostic real-time PCR for specific detection of Mycoplasma hominis DNA. Int J Microbiol 2010

17. Choe HS, Lee DS, Lee SJ, Hong SH, Park DC, Lee MK et al (2013) Performance of Anyplex II multiplex real-time PCR for the diagnosis of seven sexually transmitted infections: comparison with currently available methods. Int J Infect Dis 17(12):e1134-e1140

18. Schurch AC, Arredondo-Alonso S, Willems RJL, Goering RV (2018) Whole genome sequencing options for bacterial strain typing and epidemiologic analysis based on single nucleotide polymorphism versus gene-by-gene-based approaches. Clin Microbiol Infect 24(4):350-354

19. Smibert OC, Wilson HL, Sohail A, Narayanasamy S, Schultz MB, Ballard SA et al (2017) Donor-derived Mycoplasma hominis and an apparent cluster of $\mathrm{M}$. hominis cases in solid organ transplant recipients. Clin Infect Dis 65(9):1504-1508

20. Romero P, Munoz M, Martinez MA, Romero MI, Germain L, Maida $M$ et al (2014) Ureaplasmas and mycoplasmas in vaginal samples from prepubertal girls and the reasons for gynecological consultation. J Pediatr Adolesc Gynecol 27(1):10-13

21. McCormack WM, Almeida PC, Bailey PE, Grady EM, Lee YH (1972) Sexual activity and vaginal colonization with genital mycoplasmas. Jama. 221(12):1375-1377

22. Taylor-Robinson D, McCormack WM (1980) The genital mycoplasmas (second of two parts). N Engl J Med 302(19):1063-1067

Publisher's note Springer Nature remains neutral with regard to jurisdictional claims in published maps and institutional affiliations. 